\title{
Observations on the Springtail Leaping Organ and Jumping Mechanism Worked by a Spring
}

\author{
Seiichi Sudo ${ }^{a^{*}}$, Masahiro Shiono ${ }^{a}$, Toshiya Kainuma ${ }^{a}$, Atsushi Shirai ${ }^{b}$, and Toshiyuki Hayase ${ }^{b}$ \\ ${ }^{a}$ Faculty of Systems Science and Technology, Akita Prefectural University, Japan \\ ${ }^{\mathrm{b}}$ Institute of Fluid Science, Tohoku University, Japan
}

\begin{abstract}
This paper is concerned with a small jumping mechanism. Microscopic observations of springtail leaping organs were conducted using a confocal leaser scanning microscope. A simple springtail mechanism using a spring and small electromagnet was produced based on the observations of leaping organ and the jumping analysis of the globular springtail. Jumping characteristics of the mechanism were examined with high-speed video camera system.
\end{abstract}

Index Terms - Jumping Mechanism, Leaping Organ, Springtail, Morphology, Jumping Characteristics

\section{INTRODUCTION}

Jumping in insect movement is an effective way to escape predators, find food, and change locations. Grasshoppers, fleas, bush crickets, katydids, and locusts are particularly well known for jumping mechanisms to move around. They become airborne by jumping with a rapid extension of their legs. The jump of insects has long been regarded as a zoological problem [1].

Therefore, extensive investigations on the jump of insects have been conducted. For example, the jumping movements and performance of leafhopper insects have been analyzed from high-speed sequences of images [2]. The jumping performance and the associated leg movements as captured with high-speed imaging of small coleorrhynchan, Hackeriella veitchi, have been described [3]. The jumping mechanisms of the stick insect Timema chumash have been studied with motionscope camera software [4]. The structure of the hind limbs and the kinematics of jumping planthopper insect have been analyzed [5].

In spite of many investigations, no study has been published on the details of leaping organ and jumping characteristics of springtails except for a few papers [6]. Springtails are minute wingless arthropods that reach around $1 \mathrm{~mm}$ in size. By strictly oriented jumping, some species with a furcula are able to cover remarkable distances within a short time [7]. The furcula is normally folded forward under the abdomen, and the springtail jumps by suddenly extending the furcula ventrally and posteriorly.

* Corresponding author: Ebinokuchi 84-4, Tuchiya, Yurihonjo, Akita 015-0055, Japan, E-mail:sudo@akita-pu.ac.jp
In this paper, the springtail leaping organ was observed with confocal laser scanning microscopy. A jumping mechanism using a spring and small electromagnet was produced based on the observations of leaping organ and the jumping analysis of the springtail.

\section{EXPERIMENTAL METHOD}

\section{A. Microscopic Observations}

Globular springtails are small insects that reach around $1 \mathrm{~mm}$ in size. They have the jumping organ (furcula), which can be folded under the abdomen. Muscular action releasing the furcula can throw the insect well out of the way of predators.

In this paper, microscopic observations of the furcula were conducted using the confocal laser scanning microscope. Confocal laser scanning microscopy is fluorescence - imaging technique that produces exquisitely sharp optical sections through biological specimens. Therefore, there is a variety of applications to biology [8].

Microscopic observations were conducted using the confocal laser scanning microscope (Keyence VK9700). Test insect was in a state of suspended animation in a refrigerator. The leaping organ of the insect was observed in a short time. The resulting images can be displayed as a three-dimensional with a computer.

\section{B. Characteristic Analysis of Jumping Mechanism}

The jumping mechanism that mimicked springtail leaping organ was produced. The characteristics of the jumping mechanism was examined with high- speed video camera system. The experimental apparatus to study jumping characteristics is the same as the authors' previous paper [9]. The experimental apparatus consist of the jumping horizontal plate, the optical measurement system, and the analysis system.

The camera was set to take the picture of the twodimensional jump plate. Jumping behaviour of the mechanism on the plane was recorded with high-speed video camera system. In this experiment, frame rate of the high-speed video camera was 4,500 frames per second. The field of view the camera was the area of $485 \mathrm{~mm}$ height and $406 \mathrm{~mm}$ width. A series of frame of 
jumping behaviour of the mechanism were analyzed by the personal computer.

\section{EXPERIMENTAL RESULTS AND DISCUSSION}

\section{A. Microscopic Observations of Globular Springtail}

As was stated previously, springtails have the jumping organ (furcula), which can be folded under the abdomen, which it engages with a catch [10]. Muscular action releasing the furcula can throw the springtail well out of the way of predators. The springing organ of Tomocerus Longicornis was already drawn in lateral and ventral views [6]. In this paragraph, microscopic observation of the globular springtail, Bourletiella hortensis, was conducted using the confocal laser scanning microscope.

Figure 1 shows the laser scanning micrograph in ventral view and the two-dimensional display of surface measurement. In this experiment, test springtail in the state of apparent death by the low temperature caused by the refrigerator was observed. In Fig.1, the furcula is folded under the abdomen. The surface shapes along three lines on the micrograph are shown below. The height of the furcula from the penumbra is approximately $81 \mu \mathrm{m}$ on the line LM1, $90 \mu \mathrm{m}$ on the line LM2, and $52 \mu \mathrm{m}$ on the line LM3. Figure 2 shows the three-dimensional display of measurement result for the ventral side surface of the springtail. The biforked furcula is recognized clearly in Fig.2. V-shaped structure in Fig. 2 is furcula.

Figure 3 shows also the ventral view of the abdomen. In Fig.3, the furcula is extended. The ventral groove and the hamula are seen. The furcula is normally stowed away in the groove on underside of the abdomen. When disturbed, the hamula releases the furcula, which strikes the ground, causing the springtail to jump up to 100 times its body length into the air. The depth of the groove is approximately $46 \mu \mathrm{m}$ on the line LM2, and 54 $\mu \mathrm{m}$ on the line LM3 in Fig.3.

Figure 4 shows the three-dimensional description of the shape measurement for the ventral groove and the hamula of the springtail. Triangle-shaped valley structure in Fig.4 is the ventral groove. The furcula is folded in this ventral groove. The small peak of the neighborhood of triangular base is the hamula. This conveniently engages into the notch between the two arms of the fork whenever it is folded.

Figure 5 shows the micrograph of the furcula. The furcula of springtail consists of three segments. The length of the tip part like the blade is $69.1 \mu \mathrm{m}$. The length of bi-forked part is $229.8 \mu \mathrm{m}$ as shown in Fig.5. These two parts strike the ground, and the springtail can generate the jumping force as the reaction. At the microscopic level the furcula is clothed in minute hairs measuring 14 52 $\mu \mathrm{m}$ in length. This structure improves air flow between the furcula and the ground during take-

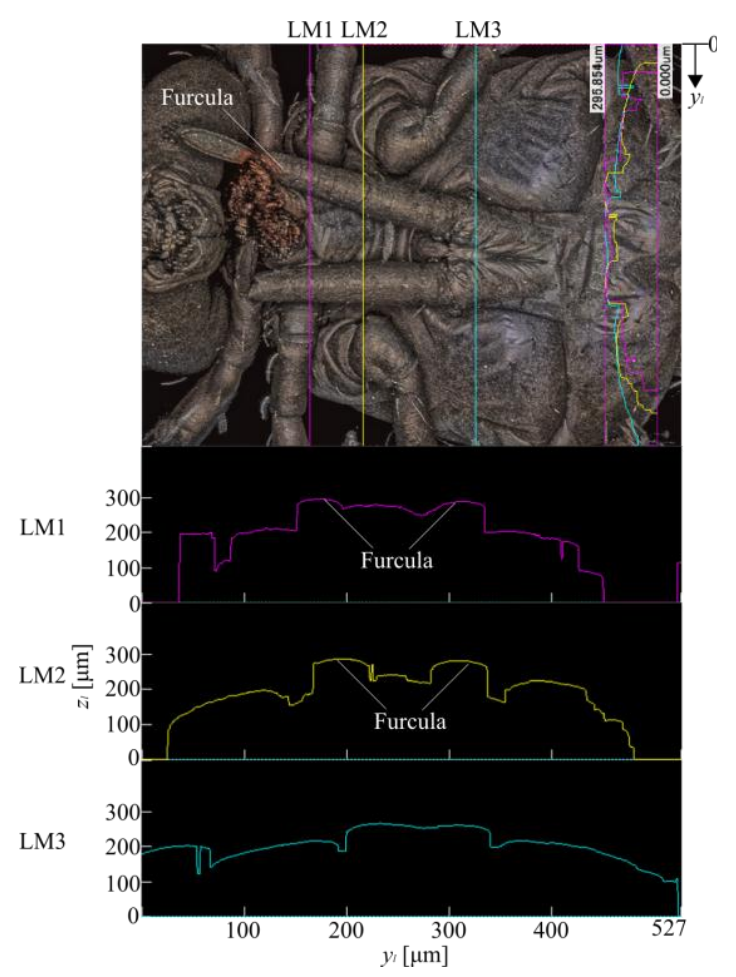

Fig. 1. Laser scanning micrograph and surface measurement in three parts of the springtail.

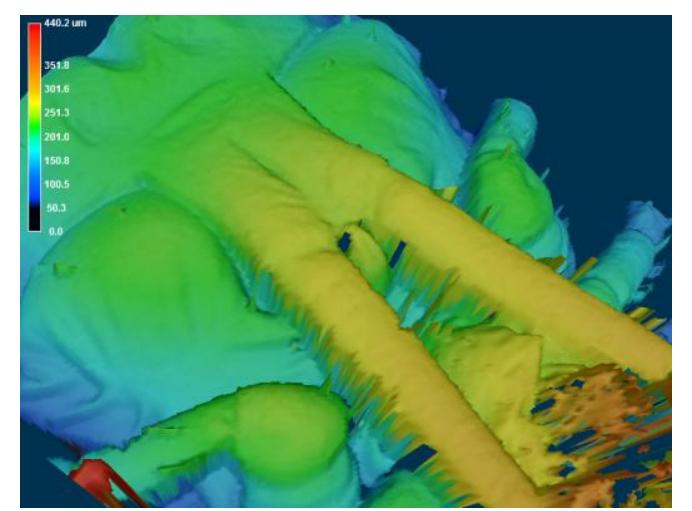

Fig. 2. Three-dimensional description of the shape measurement for the furcula.

off phase in the springtail jump [6]. Any modification of the furcula surface increases air resistance within the boundary layer will promote laminar flow and prevent the formation of turbulent eddies. Furthermore, the friction coefficient between the furcula and the board surface influence significantly the ability of the springtail to generate a thrust force.

\section{B. Microscopic Observations of Cylindrical Springtail}

Springtails are a widely distributed and highly abundant group of entognathous wingless hexapods. Commonly known as springtail, the order Collembola contains 18 families and 6500 species [10]. New species of Collembola have been being found all over the world and reported [11-13]. There are two kinds of body shape springtails. One is oval or globular springtail 


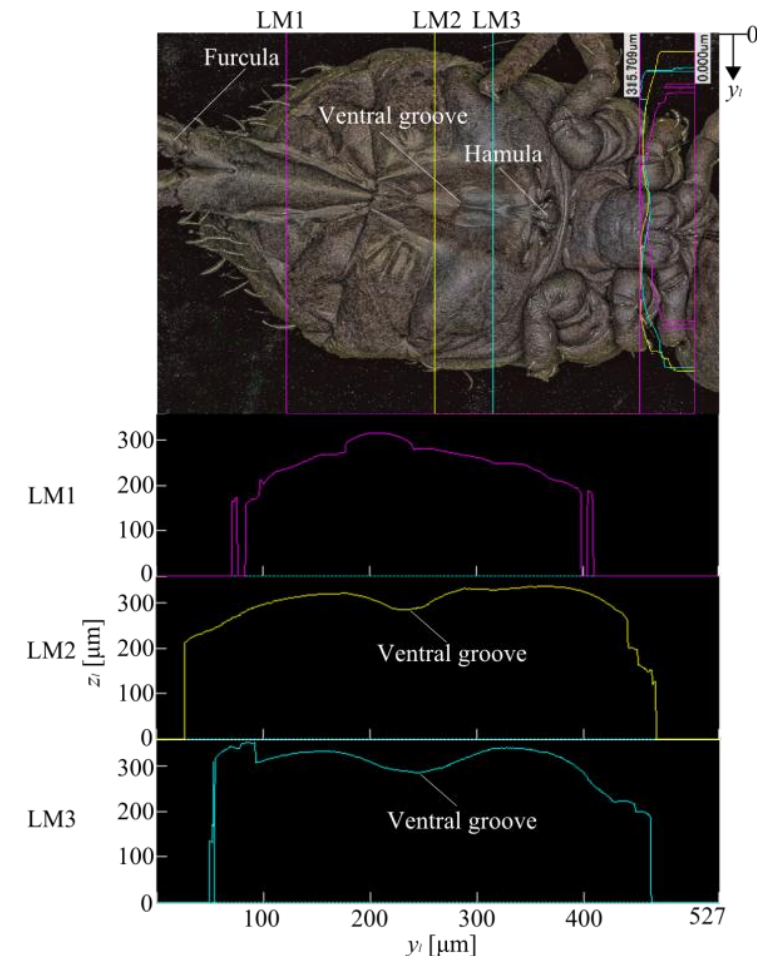

Fig. 3. Laser scanning micrograph and surface measurement in three parts of the springtail.

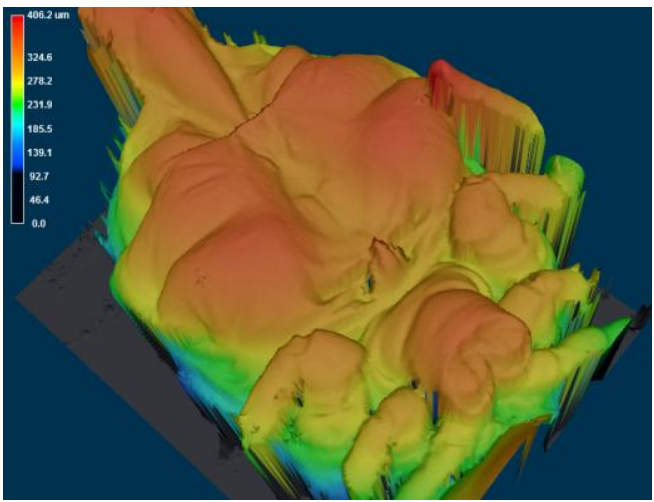

Fig. 4. Three-dimensional description of the shape measurement for the ventral groove.

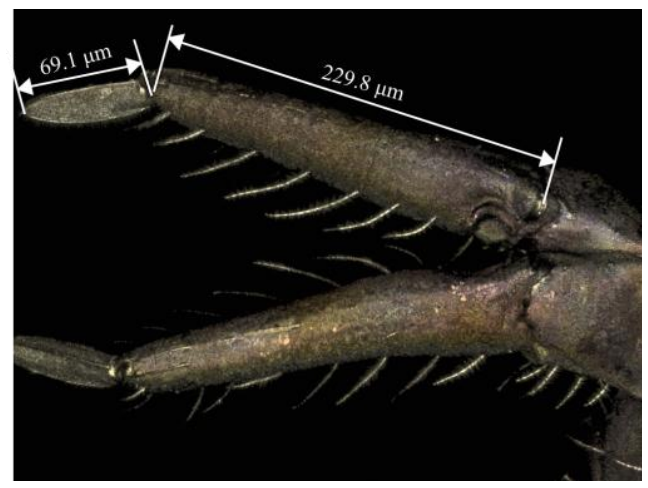

Fig. 5. Micrograph of the furcula in the extended position.

described in previous paragraph. Another is elongate or cylindrical springtail.

Figure 6 shows the cylindrical springtail collected in the field of Akita, Japan. The body length of this

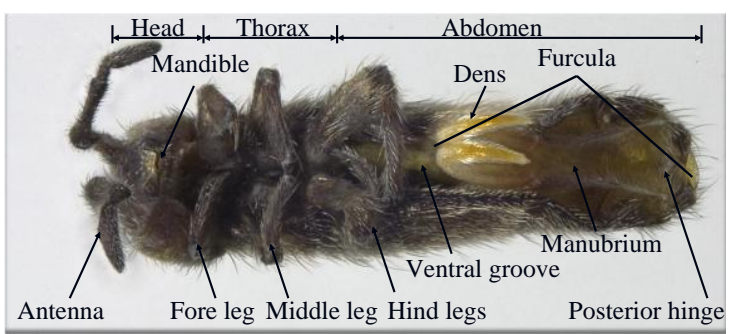

Fig. 6. Photograph and external anatomy of cylindrical springtail.
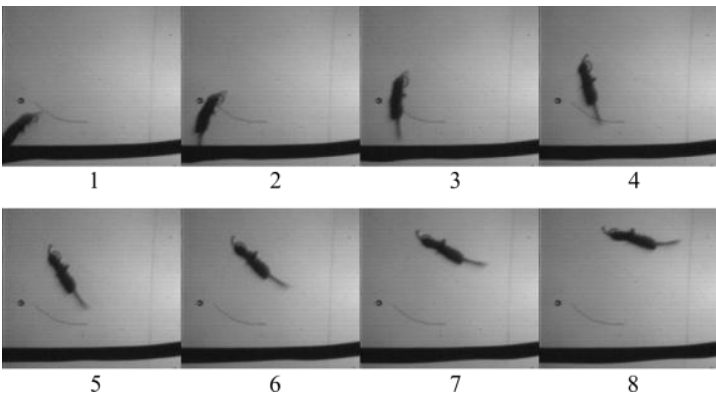

Fig. 7. Sequence of the springtail jumping from a water surface.

cylindrical springtail is $1.7 \mathrm{~mm}$. This small hexapod has a structure called a ventral tube on the underside of the abdomen. It can be seen from Fig.6 that the springtail has the jumping organ (furcula), which can be folded under the abdomen, where it engages with a catch. Details of the springtail organ of the cylindrical springtail Tomocerus longicornis has been reported [6].

The springtail showed in Fig.6 can leap from the water free surface. Figure 7 shows the jumping behaviour of the springtail from the water surface in the rectangular container. The time interval between successive images in Fig. 7 is $\delta_{t}=1.77 \mathrm{~ms}$. The black horizontal lines in the pictures show the meniscus. The selected frames from the movie prove the rotational motion of springtail body during the jump. In this experiment, the rotational direction during the jump of globular and cylindrical springtail was a backward turn. This rotational direction is different from the results in the previous paper [14]. In this paper, the springtail mechanism was produced based on the observations of leaping organ in springtails and the jumping analysis.

\section{JUMPING MECHANISM}

Recently, the mechanical leaper (Roboleaper) actuated by heating the latch with a soldering iron was constructed [15], and the study on modeling and simulation of jumping mechanism of a biomimetic robot (Flea Jumping Mechanism) was reported [16].

As was stated previously, the springtail uses its spring organ called "furcula "in emergencies. The furcula is normally stowed away in the ventral groove on the underside of the abdomen, where it engages with a catch. The furcula consists the main part of the organ, called the manubrium, and a pair of structures, called dentes (singular dens). Muscular action releasing the furcula can throw the springtail well out of the way of 
Extended state

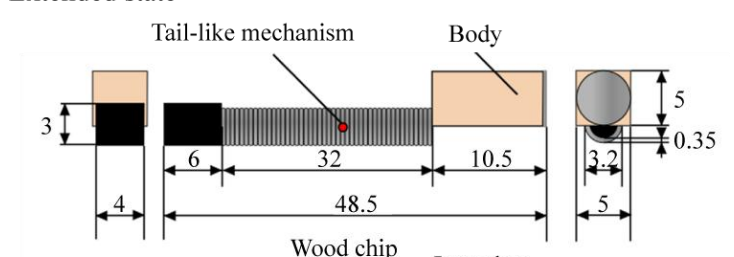

Flexed state

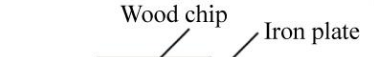

Spring

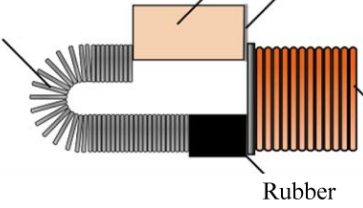

Electric magnet

Unit: $\mathrm{mm}$

Fig. 8. Drawings of the jumping mechanism in the extended and flexed states.

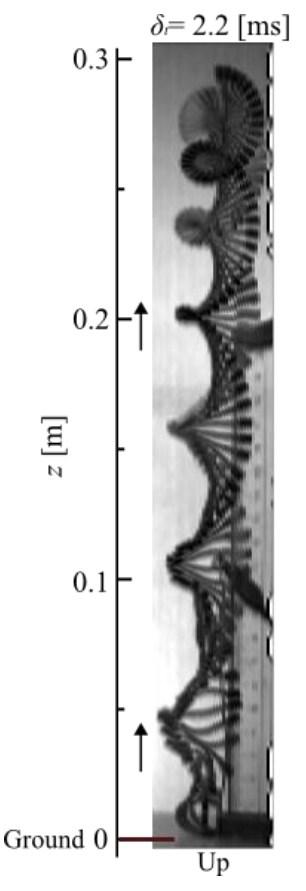

(a) Photographs

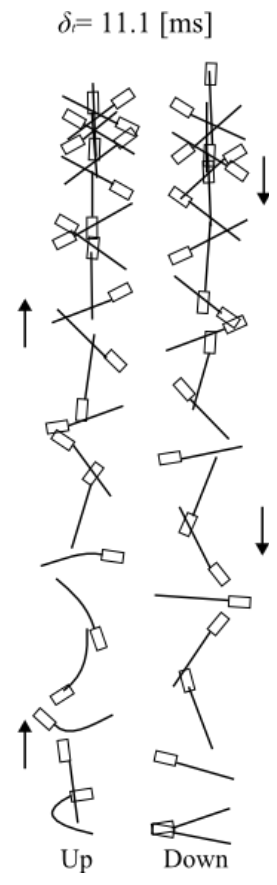

(b) Sketches
Fig. 9. Time plots in the springtail mechanism body during vertical jumping.

predators such as ants, opiliones, and pseudoscorpions. Jumping of springtails is performed by storing energy in the muscle. The elastically-stored energy in the muscle is converted into kinetic energy for jumping.

In this paper, based on the observations of leaping organ and the jumping analysis of the springtail, the jumping mechanism using the spring was constructed to be about 48 times larger than its natural counterpart. Leaping of the jumping mechanism is performed by the elastically-stored energy in bending the coil spring. Figure 8 shows the springtail mechanism in the extended and flexed states. It consists of a spring, wood chip, rubber, and iron plate with a latch that is engaged by electric magnet and released by the switch-off. The mass of the mechanism is $0.69 \mathrm{~g}$. As the ends of the spring are released, the rubber strikes the ground, catapulting the jumping mechanism upward. The bending energy stored in the spring is converted into the kinetic energy of the mechanism.

Figure 9 shows the jumping trajectories of the mechanism. The sequence of events during a jump of the jumping mechanism is shown in Fig.9 (a) by the multiple photograph method. Fig.9 (b) shows how a change of body shape can radically after the jump in jumping mechanism. The spring of jumping mechanism shows the vibration during the initial state of jumping. In this experiment, the take-off of the mechanism was almost vertical. The maximum height in the mechanism jump was $H_{m}=0.30 \mathrm{~m}$, the take-off velocity was $V=2.34$ $\mathrm{m} / \mathrm{s}$, and acceleration was $d^{2} z / d t^{2}=899 \mathrm{~m} / \mathrm{s}^{2}$. The time required to reach the maximum height was $t_{a}=0.249 \mathrm{~s}$, and the time required to reach the ground from the maximum height was $t_{d}=0.247 \mathrm{~s}$.

In this experiment, the jumping force of the mechanism was measured by the digital force gauge. The jumping force of the mechanism was $F_{\text {mech }}=1.18 \mathrm{~N}$, therefore the jumping force per unit mass was $F_{\text {mech }} / m_{\text {mech }}=1.71 \times 10^{3} \mathrm{~N} / \mathrm{kg}$. On the other hand, the jumping force per unit mass of the globular springtail was $F_{j} / m_{s}=1.77 \times 10^{3} \mathrm{~N} / \mathrm{kg}$. Both have the same performance approximately in the jumping force. The globular springtail jumped to a height of 115times of its body length. But jumping height per body length of the machine was 6.2. This fact indicates that the springtails are the excellent jumper.

The mechanism moved in a curved path. This is very common form of motion, that is, projectile motion. It can be seen from Fig.9 that the mechanism rotates like a real springtail. The direction in body rotation of the mechanism showed the backward turn. This is the same direction as the body rotation in the springtail jumping observed in the experiment. Furthermore, the mechanism showed approximately vertical leap like a real jumping springtail.

\section{CONCLUSIONS}

Microscopic observations of the springtail leaping organ were carried out with the confocal laser scanning microscope. Based on the observations of leaping organ and jumping behaviour of springtails, the jumping mechanism using a spring was designed and produced. The results obtained are summarized as follows:

(1) The structural properties of the furcula were revealed through measurements of morphological parameters. The furcula consists of posterior hinge and two joints. The first segment in the furcula is about 142 $\mu \mathrm{m}$ in length, and the length of manubrium is about 273 $\mu \mathrm{m}$. The length of dens is about $101 \mu \mathrm{m}$. The furcula is clothed in minute hairs.

(2) Springtails rotated during their leaping. The direction in body rotation was the backward turn.

(3) The jumping mechanism propelled by the bending energy stored in the spring can jump vertically. It is 
capable of jumping up to $0.3 \mathrm{~m}$. The maximum jump height is approximately 6.2 times of the mechanism body length.

\section{ACKNOWLEDGMENT}

This work was partly supported by JSPS KAKENHI Grant Number 22560173.

\section{References}

[1] Bennet-Clark, H.C., and Lucey, E.C.A. The Jump of Flea: A Study of the Energetics and a Model of the Mechanism, Journal of Experimental Biology, Vol.47 (1967), pp.5976.

[2] Burrows, M., Kinematics of Jumping in Leafhopper Insects (Hemiptera, Auchenorrhyncha, Cicadellidae), Journal of Experimental Biology, Vol.210 (2007), pp.3579-3589.

[3] Burrows, M., Hartung, V. and Hoch, H., Jumping Behaviour in a Gondwanan Relict Insect (Hemiptera: Coleorrhyncha : Peloridiidae), Journal of Experimental Biology, Vol.210 (2007), pp.3311-3318.

[4] Burrows, M., Jumping in a Wingless Stick Insect, Timema chumash (Phasmatodea, Timematodea Timematidae), Journal of Experimental Biology, Vol.211 (2008), pp.1021-1028.

[5] Burrows, M., Jumping Performance of Planthoppers (Hemiptera, Issidae), Journal of Experimental Biology, Vol.212 (2009), pp.2844-2855.

[6] Brackenbury, J. and Hunt, H., Jumping in Springtails: Mechanism and Dynamics, Journal of Zoology London, Vol.229 (1993), pp.217-236.
[7] Zettel, J., Zettel, U. and Egger, B., Jumping Technique and Climbing Behaviour of the Collembolan Ceratophysella sigillata (Collembola: Hypogastruridae), European Journal of Entomology, Vol.97(2000), pp.4145.

[8] Amos, W.B. and White, J.G., How the Confocal Laser Scanning Microscope entered Biological Research, Biology of the Cell, Vol.95 (2003), pp.335-342

[9] Sudo, S., Sekine, K., Shimizu, M., Shida, S., Yano, T. and Tanaka, Y., Basic Study on Swimming of Small Aquatic Creatures, Journal of Biomechanical Science and Engineering, Vol.4 (2009), pp.23-36.

[10] McGavin, G.C., Insects, (2000), pp/207-209, Dorling Kindersley, Limited.

[11] Beruete, E., Baquero, E. and Jordana, R., New Species of Pseudosinella (Collembola: Entomobryidae) from Karst Caves of the Basque Bio-speleologic District, Annales de la Societe Emtomologique de France, Vol.38 (2002), pp.385-398.

[12] Martinez, M., Baquero, E., Barranco, P., Arino, A.H. and Jordana, R., A New Genus and Species of Collembola from Caves of South Iberian Peninsula (Collembola, Poduromorpha, Onychiuridae), Zootaxa, Vol.734 (2004), pp.1-15.

[13] Jordana, R. and Baquero, E., A New Species of Entomobrya from Iraq (Collembola, Entomobryidae), Soil Organisms, Vol.83 (2010), pp. 351-356.

[14] Christian, E., The Jump of the Springtails, Naturwissenschaften, Vol.65 (1978), pp.495-496.

[15] Hu, D. L., Prakash, M., Chan, B. and Bush, J. W. M., Water-Walking Devices, Experiments in Fluids, Vol.43(2007), pp.769-778.

[16] Buksh, S. R., Chen, X. and Wang, W., Study of Flea Jumping Mechanism for Biomimetic Robot Design, Journal of Biomechanical Science and Engineering, Vol.5(2010), pp.41-52. 